ORIGINAL ARTICLE

\title{
Left ventricular systolic function and diastolic filling after intermittent high intensity team sports
}

\author{
K P George, E Dawson, R E Shave, G Whyte, M Jones, E Hare, D Gaze, P Collinson
}

Br J Sports Med 2004;38:452-456. doi: 10.1136/bjsm.2003.004788

See end of article for authors' affiliations ......................

Correspondence to: Dr George, Centre for Sport and Exercise Science, Liverpool John Moores University, Trueman Street, Liverpool L3 2ET, UK; k.george@ livjm.ac.uk

Accepted 20 May 2003

\begin{abstract}
Background: Prolonged steady state exercise can lead to a decrease in left ventricular (LV) function as well as promote the release of cardiac troponin $\mathrm{T}(\mathrm{cTnT})$. There is limited information on the effect of intermittent high intensity exercise of moderate duration.

Objectives: To determine the effect of intermittent high intensity exercise of moderate duration on LV function.

Methods: Nineteen male rugby and football players (mean (SD) age 21 (2) years) volunteered. Assessments, before, immediately affer, and 24 hours after competitive games, included body mass, heart rate (HR), and systolic blood pressure (sBP) as well as echocardiography to assess stroke volume (SV), ejection fraction (EF), systolic blood pressure/end systolic volume ratio (sBP/ESV), and global diastolic filling (E:A) as well as to indirectly quantify preload (LV internal dimension at end diastole (LVIDd)). Serum cTnT was analysed using a 3rd generation assay. Changes in LV function were analysed by repeated measures analysis of variance. cTnT data are presented descriptively.

Results: SV (91 (26) v91 (36) v 90 (35) ml before, after, and 24 hours after the game respectively), EF (71

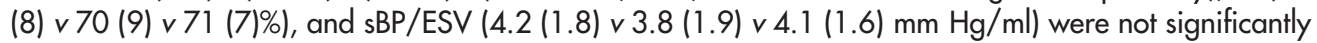
altered ( $p>0.05$ ). Interestingly, whereas LVIDd was maintained after the game (50 (5) v $50(6) \mathrm{mm}$ ), sBP was transiently but significantly reduced (131 (3) $v 122$ (3) mm Hg; $p<0.05)$. E:A was moderately $(p<0.05)$ reduced after the game $(2.0(0.4) \vee 1.5(0.4))$ but returned to baseline within 24 hours. No blood sample contained detectable levels of cTnT.

Conclusions: In this cohort, LV systolic function was not significantly altered after intermittent activity. A transient depression in global diastolic filling was partially attributable to a raised HR and could not be explained by myocyte disruption as represented by cTnT release.
\end{abstract}

$\mathrm{D}$ espite some contradictory findings, current evidence suggests that prolonged steady state exercise results in a reduction in left ventricular (LV) systolic and diastolic filling as well as occasionally promoting the appearance of cardiac specific troponins (cTnT), normally indicative of myocyte necrosis. ${ }^{12}$ Evidence of such depression in LV function and appearance of cTnT is generally transient ${ }^{34}$ and thus the term "exercise induced cardiac fatigue" (EICF) has been promoted. ${ }^{2}$ The impact on performance, the clinical consequences, and the causative factors underpinning EICF have yet to be clarified, as has the full description of the exercise circumstances that will potentially precipitate EICF.

To date most of the available EICF literature is on young, well trained athletes competing in a range of ultraendurance activities up to 24 hours in duration. ${ }^{4-14}$ Limited data are available for shorter durations (90-120 minutes) of prolonged exercise, and the few studies available are laboratory based and have produced contentious results. ${ }^{15-19}$ Owing to the extended duration of activities reported in previous studies, this type of exercise tends to be relatively submaximal and steady state in nature. Such events contain very limited periods of intense cardiovascular acceleration and/or deceleration and few or no resistive/isometric components that may increase myocardial oxygen demand.

To our knowledge, there are no data for young healthy subjects taking part in moderate duration exercise with intermittent periods of explosive, high intensity, and isometric muscle activity. Although we have some evidence that short term (15 minutes), intermittent, high intensity activity $^{19}$ and one set of resistance exercises ${ }^{20} 21$ does not reduce LV contractile function, we do not know the consequences of longer sessions of intermittent, high intensity exercise beyond the duration that has reported depressed LV function with steady state exercise. ${ }^{15-17}$ Because of the known consequences for blood pressure of isometric activity in major muscle groups ${ }^{22}$ as well as the sustained high heart rates, ${ }^{23}$ intermittent team game activities with periods of high intensity work combined with isometric muscle contractions may result in a depression in LV function after exercise and the appearance of CTnT in the same way as prolonged steady state activity.

With the high levels of participation in team sports and in the light of developing cTnT technology $y^{24}$ and debate about the clinical significance of $\mathrm{cTnT}$ in non-pathological populations, ${ }^{25}$ it is pertinent to investigate the broad concept of EICF in such circumstances. Therefore we investigated LV function and CTnT before and after common team games (rugby and football) that include a significant high intensity and/or isometric muscle contraction component.

\section{METHODS}

\section{Subjects and design}

Nineteen male subjects (mean (SD) age 21 (2) years; nine rugby league and 10 soccer players competing in interuniversity games) provided written informed consent. Exclusion criteria included any personal or early family history of

Abbreviations: $B M$, body mass; $c \operatorname{TnT}$, cardiac troponin $T ; d B P$, diastolic blood pressure; EF, ejection fraction; EICF, exercise induced cardiac fatigue; ESV, end systolic volume; HR, heart rate; LV, left ventricular; LVPWd, LV posterior free wall during diastole; LVMWS, LV meridonial wall stress; LVPWs, LV posterior free wall during systole; LVIDd, LV internal diameter during diastole; LVIDs, LV internal diameter during systole; sBP, systolic blood pressure; SV, stroke volume 
cardiopulmonary disease. Ethical approval was provided by Manchester Metropolitan University.

The study used a repeated measures design. The first echocardiographic assessment took place about 24 hours before the designated game. Immediately after the game (within 30 minutes of its completion before showering and fluid replacement), subjects were assessed again. They then returned 24 hours after the game for the final assessment. Subjects were recruited for a range of games (maximum two players for any given game) and were excluded if they had been substituted at any point of the game. Because of the multiple number of games in which the players competed, no control was placed on the nature of the opposition or the ambient conditions. An attempt to determine heart rate (HR) during games by telemetry was logistically difficult, and we resorted to a reflective interpretation of match analysis literature that suggested that both soccer and rugby included significant periods of high intensity activity interspersed with spells of low intensity (recovery) activity. ${ }^{22}$ We recorded ambient temperature and relative humidity at the assessment immediately after the game, and this varied between games (temperature range $4-12^{\circ} \mathrm{C}$; humidity range $26-54 \%$ ). All subjects were advised to abstain from hard training in the 24 hours before and after the game. They were advised not to consume alcohol or caffeine for a minimum of six hours before any assessment.

\section{Procedures}

At an initial fitness and familiarisation session, all subjects completed a general health questionnaire and were then assessed for body composition and maximum oxygen uptake $\left(\dot{\mathrm{V}}_{2} \mathrm{MAX}\right)$. Body composition was determined by anthropometry using the four site skinfold equation of Durnin and Womersley. ${ }^{25} \dot{\mathrm{V}}_{2} \mathrm{MAX}$ was determined by a continuous and progressive running treadmill exercise test using an Oxycon Alpha Sprint (Jaeger, Mijnhard bv, the Netherlands) online system.

At the main testing sessions, subjects were initially assessed for body mass (BM), with footwear, shirts, and shorts removed, on standard scales (Seca). Subjects then lay supine, and, after a 15 minute rest period, duplicate brachial artery systolic ( $\mathrm{sBP}$ ) and diastolic ( $\mathrm{dBP})$ blood pressures were assessed by standard auscultation. At this time, HR was recorded from the electrocardiograph within the echocardiography system. Each subject then underwent a two dimensionally guided $\mathrm{M}$ mode and Doppler echocardiographic examination in the left lateral decubitas position. This was conducted using an HP Sonos 1000 ultrasound imaging system with an integrated electrocardiograph (HewlettPackard, Andover, Massachusetts, USA). Using a $2.5 \mathrm{MHz}$ transducer, standard two dimensionally guided M-Mode echocardiography was used to evaluate LV structures according to ASE guidelines. ${ }^{26} \mathrm{LV}$ posterior free wall during diastole (LVPWd) and systole (LVPWs) was measured. Assessment of LV internal diameter during diastole (LVIDd) and systole (LVIDs) facilitated assessment of systolic functional indices. Ejection fraction (EF) and stroke volume (SV) were derived from $M$ mode data using the following formulae $\mathrm{e}^{27}$ :

$\mathrm{EF}(\%)=\left(\left(\operatorname{LVIDd}^{3}-\mathrm{LVIDs}^{3}\right) \times 100\right) / \operatorname{LVIDd}^{3}$

$\mathrm{SV}(\mathrm{ml})=$ LVIDd $^{3}-$ LVIDs $^{3}$

End systolic volume (ESV) of the LV was calculated as the cube of LVIDs, and thus the functional variable sBP/ESV was calculated due to its independence from LV loading. ${ }^{28}$

Doppler echocardiography was used to assess global diastolic filling. An apical four chamber view was imaged. Pulsed wave Doppler interrogation of mitral valve inflow velocities was then performed with alignment of the sample volume cursor parallel to flow at the level of the mitral annulus. Peak early filling (E wave, $\mathrm{cm}^{-1}$ ) and peak late filling (A wave, $\mathrm{cm}^{-1}$ ) velocities were measured, and the ratio of early to late diastolic filling (E:A) was calculated.

Because haemodynamic loading on the LV influences systolic function and diastolic filling indices, it was important to provide some indirect surrogate of both preload and afterload. In this respect LVIDd represented preload whereas LV meridonial wall stress (LVMWS) represented LV afterload $^{29}$ :

LVMWS $(\mathrm{g} / \mathrm{cm})=(0.334 \times(\mathrm{sBP} \times$ LVIDs $)) /(\operatorname{LVPWs}((1+$ LVPWs)/LVIDs)).

One experienced sonographer was used for all examinations. Images were recorded to high quality videotape and analysed off line by a single experienced technician. The difference in HR before and after the game made it difficult to blind the technician. A minimum of three consecutive cardiac cycles were measured and averaged.

After the echocardiographic examination, $5 \mathrm{ml}$ whole blood was drawn from an antecubital vein. This was allowed to clot, was centrifuged, and the serum drawn off and frozen $\left(-20^{\circ} \mathrm{C}\right)$ for later analysis. Serum cTnT was determined using a validated 3 rd generation assay ${ }^{30}{ }^{31}$ by electrochemiluminescence technology used in the Elecsys 1010 automated batch analyser (Roche Diagnostics, Mannheim, Germany). Quality control data for this procedure are presented elsewhere. ${ }^{31}$

\section{Data analysis}

For data analysis, the rugby and soccer players were combined into one cohort as game duration was similar and qualitative analysis of data did not reveal any different trends between the sports. Differences in BM, sBP, dBP, HR, LV systolic and diastolic filling, and LV loading before and after the game were analysed using repeated measures one way analysis of variance, with post hoc Tukey tests where appropriate. cTnT concentrations before and after the game were descriptively analysed because of the likelihood of it not being detectable at baseline. Alterations in LV function over the game were correlated with each other, $\dot{\mathrm{V}}_{2} \mathrm{MAX}, \mathrm{HR}$, and LV loading conditions by Pearson's product moment analysis. Critical $\alpha$ was set at 0.05 , and all analyses were carried out on Statistica software (Statsoft Ltd, Tulsa, Oklahoma, USA).

\section{RESULTS}

All 19 subjects played for the entire duration of the selected games. Echocardiograms were obtained for all subjects within 30 minutes of the end of play. Despite the consumption of drinks at intervals during play, BM was significantly reduced from 75.9 (5.9) to 74.8 (6.0) $\mathrm{kg}$ after the game. After 24 hours, it had increased, probably as a consequence of drinking, but had not reached baseline values (75.4 (5.8) kg). Despite this reduction in BM, there was indirect evidence of a relatively stable preload because LVIDd was not changed at any assessment point (table 1 ). A significant reduction in sBP (about $9 \mathrm{~mm} \mathrm{Hg}$ ) was reported after the game, which had almost returned to baseline levels after 24 hours of recovery. However, LVMWS, a better indicator of afterload, was not significantly reduced despite a small fall $(4 \mathrm{~g} / \mathrm{cm})$ during the game. Data for dBP were quite consistent over time $(77.8$ (1.9) $v 75.9$ (2.5) $v 78.9$ (2.6) $\mathrm{mm} \mathrm{Hg}$ ). HR immediately after the game was significantly raised in all subjects (about 23 beats/min) although this had returned to baseline after 24 hours.

Stroke volume was maintained near baseline levels in nearly all subjects, and the mean values before and after the game were not significantly different. EF, LVPWs, and sBP/ ESV-systolic functional indices more readily associated with contractility-were not different after the game. Whereas EF had decreased by about $1 \%$ immediately after the game, the 


\begin{tabular}{|c|c|c|c|}
\hline Variable & Before & After & 24 hours after \\
\hline \multicolumn{4}{|l|}{ Systolic function } \\
\hline $\mathrm{SV}$ (ml/beat) & $91(26)$ & $91(36)$ & $90(35)$ \\
\hline $\mathrm{EF}(\%)$ & $71(8)$ & $70(9)$ & 71 (7) \\
\hline $\mathrm{sBP} / \mathrm{ESV}(\mathrm{mm} \mathrm{Hg} / \mathrm{ml})$ & $4.2(1.8)$ & $3.8(1.9)$ & $4.1(1.6)$ \\
\hline LVPWs (cm) & $1.35(0.17)$ & $1.30(0.14)$ & $1.32(0.16)$ \\
\hline \multicolumn{4}{|l|}{ Diastolic function } \\
\hline$E(\mathrm{~cm} / \mathrm{s})$ & 79 (11) & $77(6)$ & $84(7)^{*}$ \\
\hline$A(\mathrm{~cm} / \mathrm{s})$ & $40(8)^{*}$ & $53(12)$ & $43(6)^{*}$ \\
\hline $\mathrm{E}: \mathrm{A}$ & $2.0(0.4)^{*}$ & $1.5(0.4)$ & $2.0(0.4)^{*}$ \\
\hline \multicolumn{4}{|l|}{ Loading } \\
\hline HR (beats/min) & $57(7)^{*}$ & $80(11)$ & $59(7)^{*}$ \\
\hline LVIDd (mm) & $50(5)$ & $50(6)$ & $50(6)$ \\
\hline $\mathrm{sBP}(\mathrm{mm} \mathrm{Hg})$ & $131(3)^{*}$ & $122(3)$ & $128(4)^{*}$ \\
\hline LVMWS $(\mathrm{g} / \mathrm{cm})$ & $110(29)$ & $106(25)$ & $108(20)$ \\
\hline \multirow{2}{*}{$\begin{array}{l}\text { Cardiac troponin } \\
\mathrm{cTnT}(\mu \mathrm{g} / \mathrm{l})\end{array}$} & & & \\
\hline & $0.00(0.0)$ & $0.00(0.0)$ & $0.00(0.0)$ \\
\hline
\end{tabular}

reduction in LVPWs was about $4 \%$ and the decline in sBP/ ESV was about $10 \%$.

Global diastolic filling, as represented by the E:A ratio, was significantly decreased immediately after the game. Whereas changes in both $\mathrm{E}$ and $\mathrm{A}$ flow velocity contributed to the altered E:A ratio, only A was significantly different (raised) after the game. Analysis of E:A data suggested a significant correlation $\left(r=-0.78, r^{2}=0.61, \mathrm{p}<0.05\right)$ between E:A and HR after the game (fig 1) but limited or no relation to other loading factors or indices of global fitness $(r=0.13-0.31$, $\mathrm{p}>0.05)$. Likewise there was no significant correlation between E:A and indices of LV contractility after the game $(r=-0.07$ to $-0.36, \mathrm{p}>0.05)$. cTnT was not detected in any blood sample.

\section{DISCUSSION}

This study is the first to attempt to document the potential for 80-90 minutes of intermittent, high intensity exercise to elicit characteristics of EICF. In so doing it adds to a growing database for prolonged endurance and ultraendurance activity that occurs predominantly at steady state exercise intensities.

The most obvious finding is that the field based exercise adopted did not result in any statistically significant evidence of depressed LV systolic function after the game. It is, however, interesting to note that SBP/ESV (a surrogate of LV contractility least affected by loading) was decreased by about $10 \%$ and LVPWs by about $4 \%$ after the game. EF was unaltered despite a significant reduction in sBP and a small fall in LVMWS. Whether these alterations are functionally significant, despite the lack of statistical significance, is

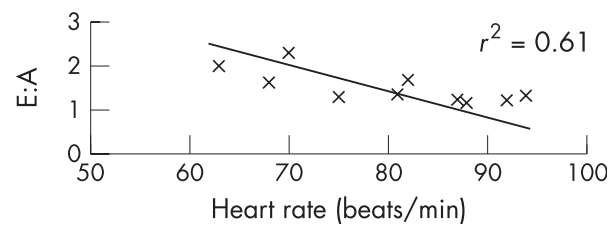

Figure 1 Relation between heart rate and ratio of early to late diastolic filling $(E: A)$ after the game. difficult to determine, as such changes are close to, or within, measurement variability. Further research with a larger sample size, using additional indices of systolic function derived by tissue Doppler, is needed to elaborate a potential LV contractile depression.

The conservative interpretation of these data is that LV contractility was not significantly altered, probably because of the relatively well maintained preload. This is probably similar to the very short duration, high intensity exercise previously studied..$^{20}$ The lack of significant changes in LV contractile function may also be due to the fact that the total external and cardiovascular workloads imposed were limited, despite high mean HRs and increased myocardial oxygen demand caused by short periods of isometric or high intensity work, compared with some previous ultraendurance activity. ${ }^{7}$ This is not surprising given the immense exercise volumes and effort required over the long hours of an Ironman triathlon ${ }^{7^{10}}$ or 24 hour runs. ${ }^{56}$ Interestingly there are few field based cardiac fatigue studies that have investigated exercise of one to two hours duration. ${ }^{12}$ The closest examples may be marathon running ${ }^{32-35}$ with race durations of three plus hours where only sporadic evidence of EICF is noted. ${ }^{34}$ Laboratory based studies with 60-120 minutes of steady state exercise have provided evidence to both support $\mathrm{t}^{15-17}$ and refute $^{1819}$ the existence of EICF. The variability in results between studies may be due to differences in exercise intensity, subject, or technical measurements.

Whether the lack of a statistically significant depression in LV systolic function is a consequence of the relatively low level of competitive activity or the subelite nature of the cohort is impossible to determine. University players may not play at the same intensity as more elite players, and they are also unlikely to be as well trained. A continuation of this work may use different cohorts or different exercise regimens. A specific area to develop from this work may be an investigation of the consequences of hard resistance training sessions on LV function after exercise. To our knowledge, no such data are available but may be interesting given the haemodynamic pressure loading on the LV.

The decreased E:A suggests some form of depression of global diastolic filling. Although diastolic filling has received less attention than systolic function, the statistical outcome 
and magnitude of change in our data (2.0 to 1.5 ) is fairly consistent with a range of previous research. ${ }^{35}$ The reduction in E:A after the game is difficult to fully explain; however, the increase in HR after the game (significantly correlated with E:A) may account for about $60 \%$ of the variability in E:A (fig 1). Somewhat surprisingly, a raised HR after exercise could not explain the reduction in E:A reported in similar research. ${ }^{3}$ Other indices of LV loading measured in our study could not fully explain the remaining variability in E:A. One potential contributory factor, myocyte necrosis, can be largely discounted because of the lack of any detectable cTnT in any subject after exercise. Any further explanation for this alteration in function is therefore speculative at best, but the disturbance of intracellular $\mathrm{Ca}^{2+}$ metabolism specifically related to its removal from the cytosol has been suggested. Further research is required to clarify the relation between HR and diastolic filling after exercise and whether this is the primary contributor to the reduction in E:A reported in most cardiac fatigue studies. It is interesting that diastolic filling decreased in the absence of a depression in systolic function. Whether diastolic filling is more sensitive to the impact of prolonged and/or intermittent exercise than systolic function is again speculative but worthy of further enquiry. It is also important to note that diastolic filling is complex and poorly understood. Furthermore, it is recognised that global indices of diastolic filling (E:A) can be quite limited, and interpretation must be made with care. $^{36}$ Future researchers may wish to use technologies such as tissue Doppler to obtain a more complete picture of the effect of prolonged exercise on LV diastolic filling.

The lack of cTnT in any blood sample suggests that this type of exercise does not result in myocyte necrosis or alterations in membrane permeability ${ }^{13}$ in this cohort. This suggests that myocardial stunning ${ }^{37}$ is not propagated within intermittent intensity exercise of 80-90 minutes duration. Blood cTnT concentrations after exercise have previously been assessed in steady state, field based research of duration greater than 180 minutes. Thus there are limited data with which to compare our results and the absence of cTnT. cTnT has been detected in longer studies, ${ }^{10}{ }^{14}$ but this finding is by no means consistent between studies or within specific populations. The predisposing factors for $\mathrm{CTnT}$ appearance, and thus the clinical significance, in healthy populations performing exercise require further study. When interpreting our cTnT data, it is important to note that a potential limitation of this study was the timing of the blood sample after exercise (within 30 minutes of cessation of exercise). The time course of pathological appearance of cTnT (about four hours) may have meant that blood sampling occurred too soon to pick up the appearance of cTnT. However, if pathological release of cTnT had occurred, it would probably have still been detectable 24 hours after exercise. Membrane leakage of the cytosolic component of CTnT, as proposed by Neumayr et al, ${ }^{13}$ may also have occurred and been missed. However, this can appear substantially earlier than in scenarios such as acute myocardial infarction. Indeed there is evidence from animal models of differential temporal release patterns for cTnT depending on its origin, with membrane damage causing functionally unbound cTnT release within minutes of reperfusion in a rat heart model. ${ }^{38}$ Unpublished observations from our laboratory have reported raised CTnT within two hours of the onset of endurance exercise in humans.

In conclusion, intermittent exercise in this cohort did not result in a significant depression in LV systolic function, although further research may be valuable to confirm this. A small but significant reduction in diastolic filling after exercise could only partially be explained by HR dynamics,
Take home message

Exercise bouts of moderate duration that contain intermittent periods of high intensity activity do not result in significant LV systolic functional alterations or evidence of myocyte necrosis. The alterations in LV diastolic filling after exercise are partially related to HR changes. All changes are normalised 24 hours after exercise.

and further research is required to understand this change. The exercise bout did not produce any evidence of cardiac myocyte necrosis after exercise.

\section{Authors' affiliations}

K P George, Liverpool John Moores University, Liverpool, UK E Dawson, M Jones, E Hare, Manchester Metropolitan University, Manchester, UK

R E Shave, G Whyte, British Olympic Medical Centre, Northwick Park Hospital, Harrow, Middlesex, UK

D Gaze, P Collinson, St George's, London, UK

\section{REFERENCES}

1 McGavock JM, Warburton DER, Taylor D, et al. The effects of prolonged strenuous exercise on left ventricular function: a brief review. Heart Lung 2002;31:279-92.

2 Dawson E, George K, Shave R, et al. Does the heart fatigue subsequent to prolonged exercise in humans? Sports Med 2003;33:356-80.

3 Lucia A, Serratosa L, Saborido A, et al. Short-term effects of marathon running: no evidence of cardiac dysfunction. Med Sci Sports Exerc 1999;31:1414-21.

4 Whyte G, George K, Sharma S, et al. Cardiac fatigue following prolonged endurance exercise of differing distances. Med Sci Sports Ex 2000;32:1067-72.

5 Niemela KO, Palatsi IJ, Ikaheimo MJ, et al. Evidence of impaired left ventricular performance after an uninterrupted competitive 24 hour run. Circ 1984;70:350-6.

6 Niemela K, Palatsi I, Ikaheimo M, et al. Impaired left ventricular diastolic filling in athletes after utterly strenuous prolonged exercise. Int J Sports Med 1987;8:61-5

7 Douglas PS, O’Toole ML, Hiller DB, et al. Cardiac fatigue after prolonged exercise. Circ 1987;76:1206-13.

8 Bonetti A, Tirelli F, Albertini R, et al. Serum cardiac troponin T after repeated endurance exercise events. Int J Sports Med 1996;17:259-67.

9 Davila-Roman VG, Guest TM, Tuteur PG, et al. Transient right but not left ventricular dysfunction after strenuous exercise at high altitude. J Am Coll Cardiol 1997;30:468-73.

10 Rifai N, Douglas PS, O'Toole M, et al. Cardiac troponin T and I, echocardiographic wall motion analyses, and ejection fractions in athletes participating in the Hawaii Ironman Triathlon. Am J Cardiol 1999;83:1085-9.

11 Haykowsky M, Welsh R, Humen D, et al. Impaired left ventricular systolic function after a half-Ironman race. Can J Cardiol 2001;17:687-90.

12 Neumayr G, Gaenzer H, Pfister R, et al. Plasma levels of cardiac troponin I after prolonged strenuous endurance exercise. Am J Cardiol 2001;87:369-71.

13 Neumayr G, Pfister R, Mitterbauer G, et al. Effect of the "Race Across The Alps" in elite cyclists on plasma cardiac troponins I and T. Am J Cardiol 2002;89:484-6.

14 Shave RE, Dawson E, Whyte G, et al. Evidence of exercise-induced cardiac dysfunction and elevated $\mathrm{cTnT}$ in separate cohorts competing in an ultraendurance mountain marathon race. Int J Sports Med 2002a;23:489-94.

15 Ketelhut R, Losem CJ, Messerli FH. Depressed systolic and diastolic cardiac function after prolonged aerobic exercise in healthy subjects. Int I Sports Med 1992;13:293-7.

16 Ketelhut $\mathbf{R}$, Losem $\mathrm{CJ}$, Messerli $\mathrm{FH}$. Is a decrease in arterial pressure during long-term aerobic exercise caused by a fall in cardiac pump function? Am Heart J 1994; 127:567-71

17 Eysmann SB, Gervino E, Vatner DE, et al. Prolonged exercise alters $\beta$ adrenergic responsiveness in healthy sedentary humans. J Appl Physiol 1996;80:16-22.

18 Upton MT, Rerych SK, Roeback JR, et al. Effect of brief and prolonged exercise on left ventricular performance. Am J Cardiol 1980:45:1154-60.

19 Palatini P, Bongiovi S, Marcor F, et al. Left ventricular performance during prolonged exercise and early recovery in healthy subjects. Eur J Appl Physiol 1994;69:396-401.

20 Foster C, Meyer K, Georgakopoulos N, et al. Leff ventricular function during interval and steady state exercise. Med Sci Sports Exerc 1999;31:1157-62.

21 Karlsdottir AE, Foster C, Porcari JP, et al. Hemodynamic responses during aerobic and resistance exercise. J Cardiopulm Rehabil 2002;22:170-7. 
22 MacDougall JD, Tuxen D, Sale DG et al. Arterial blood pressure response to heavy resistance exercise. J Appl Physiol 1985;58:785-90.

23 Reilly T. Motion analysis and physiological demands. In: Reilly T, ed. Science and soccer. London: E\&FN Spon, 1996.

24 Collinson PO, Boa FG, Gaze DC. Measurement of cardiac troponins. Ann Clin Biochem 2001;38:423-49.

25 Durnin JVGA, Womersley J. Body fat assessed from total body density and its estimation from skinfold thicknesses: measurement on 481 men and women aged 16-72 years. Br J Nutr 1974;32:77-97.

26 Sahn DJ, DeMaria A, Kisslo J, et al. Recommendations regarding quantitation in M-mode echocardiography: results of a survey of echocardiographic measurements. Circulation 1978;58:1072-83.

27 Longo MR, Guzman AW, Triebwasser JH. Normal values and commonly used echocardiographic formulae for adults. Aviat Space Environ Med 1975;46:1062-4

28 Vanoverschelde JL, Younis LT, Melin JA, et al. Prolonged exercise induces left ventricular dysfunction in healthy subjects. J Appl Physiol 1991;70:1356-63.

29 Reichek N, Wilson J, Sutton $M$, et al. Noninvasive determination of left ventricular end-systolic stress: validation of the method and initial application. Circulation 1982;65:99-108.

30 Hallermayer K, Klenner D, Vogel R. Use of recombinant human cardiac troponin T for the standardization of third generation troponin T methods. Scand J Clin Lab Invest 1999:59:128-31.
31 Shave R, Dawson E, Whyte G, et al. The cardiospecificity of the thirdgeneration cTnT assay after exercise-induced muscle damage. Med Sci Sports Exerc 2002b;34:651-4.

32 Ohman EM, Teo KK, Johnson AH, et al. Abnormal cardiac enzyme responses after strenuous exercise: alternative diagnostic aids. BMJ 1982;285:1523-6.

33 Perrault $\mathrm{H}$, Peronnet F, Lebeau R, et al. Echocardiographic assessment of left ventricular performance before and after marathon running. Am Heart $J$ 1986;112:1026-31.

34 Manier G, Wickers F, Lomenech AM, et al. Echocardiographic assessment of myocardial performance after prolonged strenuous exercise. Eur Heart $J$ $1991 ; 12: 1183-8$.

35 Siegel AJ, Lewandrowski EL, Chun KY, et al. Changes in cardiac markers including B-natriuretic peptide in runners after the Boston marathon. Am J Cardiol 2001;88:920-3.

36 Libonati JR. Myocardial diastolic filling and exercise. Med Sci Sports Exerc 1999;31:1741-7.

37 Rowe WJ. Endurance exercise and injury to the heart. Sports Med 1993;16:73-9.

38 Remppis A, Scheffold T, Greten J, et al. Intracellular compartmentation of troponin T: release kinetics after global ischemia and calcium paradox in the isolated perfused rat heart. J Mol Cell Cardiol 1995;27:793-803.

\section{ELECTRONIC PAGES}

\section{BJSM Online case reports: http://bjsm.bmijournals.com/}

7 con

he following electronic only articles are published in conjunction with this issue of BJSM.

The reliability of team-based primary data collectors for the collection of exposure and protective equipment use data in community sport

R A Braham, C F Finch

Objective: Reliable data allows for the generalisation of study findings to the wider population. The aim of this study was to assess the reliability of using community team based primary data collectors for the collection of exposure and protective equipment use data.

Methods: Nine clubs (23 teams) from a metropolitan Australian Football league in Victoria each provided one primary data collector to monitor exposure and protective equipment use over a regular playing season. Four random audits of this data collection for each team were conducted throughout the regular playing season. The audits were compared with data collected by the club data collectors and the level of agreement assessed.

Results: Although exposure data agreement was higher during competition and protective equipment use agreement higher during training, there was no significant difference in data collected by the primary data collectors and the random audits.

Conclusions: The use of trained data collectors associated with Australian Football teams provides reliable information about player exposure and protective equipment use in community intervention studies.

(Br J Sports Med 2004;38:e15) http://bjsm.bmjjournals.com/ cgi/content/full/38/4/e15

\section{The dipsomania of great distance: water intoxication in an Ironman triathlete}

T D Noakes, K Sharwood, M Collins, et al

Of 371 athletes (62\% of all finishers) whose weights were measured before and after the $226 \mathrm{~km}$ South African Ironman Triathlon, the athlete who gained the most weight $(3.6 \mathrm{~kg})$ during the race was the only competitor to develop symptomatic hyponatraemia. During recovery, he excreted an excess of 4.6 litres of urine. This case report again confirms that symptomatic hyponatraemia is caused by considerable fluid overload independent of appreciable $\mathrm{NaCl}$ losses. Hence prevention of the condition requires that athletes be warned not to drink excessively large volumes of fluid (dipsomania) during very prolonged exercise. This case report also shows that there is a delayed diuresis in this condition and that it is not caused by renal failure.

(Br J Sports Med 2004;38:el6) http://bjsm.bmjjournals.com/ cgi/content/full/38/4/el6 